Le genre de la maladie : pratiques, discours, textes et représentations

\title{
Le genre de la maladie : pratiques, discours, textes et représentations
}

Sibylle GOEPPER et Emmanuelle ROMANET

\section{(2) OpenEdition \\ Journals}

Édition électronique

URL : http://journals.openedition.org/transtexts/644

DOI : $10.4000 /$ transtexts. 644

ISSN : 2105-2549

\section{Éditeur}

Gregory B. Lee

\section{Référence électronique}

Sibylle GOEPPER et Emmanuelle ROMANET, « Le genre de la maladie : pratiques, discours, textes et représentations », Transtext(e)s Transcultures 跨文本跨文化 [En ligne], 11 | 2016, mis en ligne le 29 juin 2017, consulté le 25 septembre 2020. URL : http://journals.openedition.org/transtexts/644 ; DOI : https://doi.org/10.4000/transtexts.644

Ce document a été généré automatiquement le 25 septembre 2020.

(c) Tous droits réservés 


\title{
Le genre de la maladie : pratiques, discours, textes et représentations
}

\author{
Sibylle GOEPPER et Emmanuelle ROMANET
}

1 En 2013 paraissait sous la direction de Nicole Edelman et de Florence Rochefort un numéro de la revue Clio intitulé «Quand la médecine fait le genre $»^{1}$ qui attestait du dynamisme de la recherche française consacrée la relation entre genre, médecine et santé. Le premier colloque international à s'être tenu en France sur le thème " Genre et Santé » à l'Université Diderot à Paris témoignait, en 2015, du même intérêt. Si ces travaux ont mis en lumière un large champ de recherche, ils ont aussi permis de constater que la dimension des représentations, telles que les proposent entre autres la littérature et les arts, n'étaient que très peu prise en compte lorsqu'il s'agissait d'aborder ces problématiques.

2 La journée d'études organisée par le groupe Genre Lyon 3 (GL3) le 13 novembre 2015 à l'Université Jean Moulin Lyon 3 sur le thème " Le genre de la maladie : textes, discours et représentations " se proposait de contribuer à combler cette lacune. Sa démarche était double : d'un côté elle intégrait la littérature et les arts à la réflexion, en partant du principe que ces domaines, aux côtés de l'histoire et des sciences sociales, aidaient à affiner la perception et la compréhension culturelle du phénomène de la maladie et des liens qu'il entretient avec le genre; de l'autre, elle s'appuyait sur une perspective transdisciplinaire, transculturelle et diachronique.

3 Dès lors en effet que l'on considère les genres comme des identités construites à la fois structurellement et relationnellement, qui se négocient et se réalisent dans un contexte et un environnement précis, quitter la période contemporaine et/ou le territoire français paraît particulièrement prometteur. Le regard posé sur d'autres époques, cultures et langues favorise en outre per se les croisements et carrefours, autrement dit l'intersectionnalité nécessaire afin d'éviter le piège de l'essentialisation de l'« homme », de la « femme » et de leur relation à la maladie.

4 Mais quelle peut être précisément la contribution de la littérature et des arts lorsqu'il s'agit d'analyser l'impact des identités et des dynamiques sociales genrées sur la santé des individus? Les phénomènes de différenciation entre hommes et femmes, qui sont 
assurément le résultat d'un rapport de force et de domination, sont d'une part reflétés par les représentations que véhiculent les champs artistiques. À bien des égards, ces disciplines constituent en effet un prolongement, certes littérarisé, médiatisé, mais néanmoins vraisemblable des représentations de la maladie ayant cours dans le réel des sociétés, des collectifs, des familles. Là (ré)émergent les croyances partagées sur ce qu'est ou doit être un homme ou une femme et donc sur ce qu'est et doit être sa maladie. Mais leur rôle ne s'arrête pas là, car si les représentations peuvent venir renforcer ces récits, elles sont également à même de les compléter, de les amplifier ou de les nuancer. Plus encore: parce qu'elles créent par le biais de la fiction et de l'imaginaire un espace paradoxal où tout est à la fois absolument vrai et absolument faux, elles sont sans nul doute aussi en mesure de bousculer le discours genré sur la maladie. L'œuvre, quand elle transcende les visions et conceptions habituelles devient ainsi le lieu d'une (re)construction capable de contester les hiérarchies de genre, de les modifier et, pourquoi pas, de les abolir.

5 Le présent numéro de la revue Transtext(e)s Transcultures. Journal of Global Cultural Studies rassemble le fruit du travail mené autour de ces différentes questions par des spécialistes d'histoire et de littérature française, germanique, hispanique, grecque, néohellénique, anglaise et chinoise. Il se caractérise d'abord par la diversité des approches et des sources. Ces dernières relèvent du domaine médical (cours et traités de médecine), mais également de la sphère privée (correspondances) et même juridique (procès-verbaux de tribunaux). Les textes littéraires abordés appartiennent quant à eux à la poésie, à la nouvelle et au roman. On y trouve aussi des genres intermédiaires, comme des récits de diagnostics, s'inscrivant à la fois dans le discours médical et la tradition littéraire, ou le métadiscours de la critique littéraire. Le pont jeté entre les lieux et les époques relie la Grèce antique à la Grèce moderne, le Saint-Empire Romain germanique du XVIIe siècle à l'Allemagne réunifiée des années 2000, l'Espagne à la charnière entre XVIIIe et XIXe siècle, la France des XIXe et XXe siècles, tout en passant par la Chine des années 1920 et les États-Unis de l'avant Seconde Guerre mondiale. Pathologies obstétriques et gynécologiques, syphilis, hystérie, «folie furieuse ", schizophrénie, autisme, narcissisme, névrose, chagrin d'amour, accident cérébral, encéphalite ou encore addiction à l'alcool: les maux parcourus aussi sont multiples. Physiques, psychiques ou moraux, ils correspondent à des douleurs réelles ou à des stigmates apposés par la société à différentes époques et permettent de questionner l'impact individuel et collectif de la maladie à travers le genre des soigné.e.s et des soignant.e.s.

6 Le croisement des archives historiques et des œuvres artistiques confirme la vision d'une médecine qui, loin d'être neutre, a de tout temps été à la fois représentative et pourvoyeuse de systèmes interprétatifs. Comme le fait observer Nicole Edelman, celleci participe "à l'élaboration de normes biologiques, éthiques ou politiques qui ont validé des hiérarchies entre les sexes ». Ainsi le corps féminin est-il presque toujours pensé par référence au corps masculin, et c'est l'imperfection ou le manque qui le caractérise.

7 Chacun à leur manière, Pascal Luccioni et Sylvie Imparato-Prieur analysent la naturalisation, la différenciation et l'infériorisation dont le féminin a fait l'objet à travers les époques. Nous parlons ici à dessein de «féminin » et non de «femmes » car les hommes ne sont pas épargnés par les injonctions de genre lorsqu'ils ne correspondent pas au modèle masculin hégémonique de virilité, de bravoure et de 
performance. Ces deux contributions révèlent le rôle de régulateur social que revêt le discours médical qui, en s'appuyant sur la légitimité d'une démarche soi-disant scientifique, tend à ériger l'ordre établi en un ordre naturel, et donc incontournable. Sous couvert de valorisation de l'essence de «la » femme, la parole des médecins relaie la volonté idéologique qui cherche à instrumentaliser les corps au nom du bien commun. Les femmes se voient ainsi refuser certaines unions, permises aux hommes, ou alors confisquer leur utérus, objet constant à la fois de fascination et de répulsion, en se voyant contraintes à la maternité. Ce phénomène n'est du reste pas propre à l'Europe. Jacqueline Estran explique en effet comment, en Chine, les principes du Yin et du Yang, qui complètent la dimension biologique du discours médical, concourent euxaussi à renforcer la domination de genre. Sont ainsi mis en lumière des mécanismes d'éviction qui éloignent les femmes des instances de savoir et de l'espace public, mécanismes qui demeurent actifs jusqu'à nos jours.

Le regard porté sur l'enjeu politique et économique que constitue le contrôle de la démographie permet cependant aussi de prendre conscience de la profonde ambivalence du lien entre genre, maladie et pouvoir. Car s'il est indéniable que le discours médical empêche les femmes de disposer librement de leur corps et les incite à se conformer aux attentes de la société, il contribue dans le même temps à des progrès tangibles dans la connaissance de leurs organes spécifiques, de même qu'il dicte la mise en place de politiques de santé publique qui constituent bel et bien une avancée. C'est ce qu'établissent, entre autres, les recherches d'Emmanuelle Romanet-Da Fonseca.

Les discours sur les «maladies de femmes » sont principalement tenus par les hommes, élément sans nul doute constitutif de leur relation inégalitaire. Or, là encore, il y a ambivalence. Celle-ci se situe au niveau des patient.e.s et des soignant.e.s et prouve que les constellations "médecins/masculins/dominants " versus «malades/féminines/ dominées " sont plus complexes qu'il n'y paraît à première vue. Stéphanie ChapuisDesprés explique en effet comment voisines, matrones et sages-femmes ont tout à la fois pour fonction de protéger les parturientes, mais aussi de les surveiller. Leur participation au système de contrôle du corps des femmes par la société pose la question de l'intériorisation de la norme. La littérature fait de son côté la part belle aux personnages d'épouses et d'infirmières sacrificielles qui semblent avoir intégré les attendus de la société envers leur "genre ». Or Catherine Delesalle-Nancey insiste sur la réelle complémentarité des êtres qui forment le couple soignant/soigné. Surtout, elle rappelle que sous les traits des femmes dévouées mises en scène chez Fitzgerald se cache l'auteur en personne, autrement dit un homme, qui se consacra toute sa vie à son épouse malade. Le schéma selon lequel les femmes seraient en charge des soins tandis que les hommes en bénéficieraient, avec ce que cette relation suppose de dissymétrie, s'en trouve relativisé.

10 Les réflexions proposées corroborent par ailleurs le caractère profondément mouvant des discriminations de genre. La reconfiguration des relations de domination et de hiérarchie dépend d'un côté des découvertes et des nouvelles technologies qui modifient, de façon plus ou moins abrupte, le discours médical sur la différence des sexes; de l'autre, de l'évolution des argumentaires façonnés par les pouvoirs politiques, économiques, culturels et sociaux. L'influence exercée par toute une série d'autres facteurs que celui du progrès médical apparaît clairement à la lecture du dossier. Parmi ces facteurs, on peut citer les rivalités entre les différentes professions du champ médical (médecins, chirurgiens, sages-femmes), les rapports de classe, qui entraînent 
des disparités de rémunération pour les soins apportés entre autodidactes et érudits ou un déficit d'information sur la maladie quand on est issu du peuple et des campagnes et non de la bourgeoisie des villes. Signalons pour finir l'importance croissante de l'interaction avec la sphère judiciaire au fur et à mesure que les malades vont toujours plus souvent en justice afin d'obtenir réparation pour le tort subi.

11 Si l'on peut estimer que les inégalités sont toujours reconstruites et qu'elles constituent en ce sens un invariant des sociétés, les arts semblent offrir un lieu propice afin de porter un regard moins normatif sur les corps et leur sexuation. Sophie Coavoux montre comment le genre poétique permet de détourner et de subvertir les logiques pseudo-médicales qui attribuent les catégories de "honteux » et de "vertueux », de "sain " et de "malsain", de "pur» et d'« impur» en fonction de la seule norme hétérosexuelle, tandis que dans le domaine de la prose, Sibylle Goepper donne à entendre des voix alternatives et dissonantes au sein d'une société majoritaire et d'un monde médical qui divisent trop nettement les êtres humains en deux sexes. Toutes deux abordent des œuvres qui proposent de penser le genre et la sexualité en dehors de l'hétéronormativité. C'est dans cette reconfiguration du réel, rendue possible par la translation artistique, autrement dit par le symbolique, les images et les métaphores, que peut avoir lieu la prise de conscience et la remise en question des discriminations. En révélant une infinité de masculins et de féminins, les esthétiques analysées minent les hiérarchies existantes et favorisent le dépassement de la pensée binaire. C'est enfin sur le plan de l'écriture même que la maladie semble en mesure de faire émerger de nouvelles potentialités. Telle est la dimension qu'éclaire Thu Thuy Bui en étudiant un matériau langagier et textuel qui, par la rage et la folie qui l'habitent, constitue en soi un lieu de résistance face à la douleur infligée par le deuil et l'exil. Cette thérapeutique des «mots écrits » face aux «maux vécus » est l'antidote élaboré afin d'échapper à la déraison. Et elle est tout à la fois le moyen et la finalité de la quête identitaire de l'auteure à travers ses personnages.

12 Le présent dossier accueille dix contributions réparties en trois parties qui vont de l'Histoire aux représentations artistiques.

Dans son introduction, Nicole Edelman se propose de répondre à la question : « Que fait le genre à la maladie ? » Pour ce faire, elle se positionne sur le terrain de l'histoire de la médecine, tout en rappelant d'emblée que le genre est une façon de penser le monde qui permet « de saisir toutes les formes de domination entre hommes et femmes et plus largement entre tous les êtres humains ». L'auteure interroge également de façon critique les catégories de sexe masculin et féminin dont la binarité a été problématisée par toute une série de découvertes biologiques et médicales. Elle retrace ensuite l'évolution de la conception genrée du corps et de ses pathologies depuis la Grèce antique jusqu'à nos jours, en passant par les césures majeures que constituent la découverte de la cause physiologique de la maladie à la fin du XVIIIe, celle du « milieu intérieur » et de l'inconscient à la fin du XIXe siècle. Ce panorama lui permet d'établir que la médecine a constamment participé de la hiérarchisation entre les genres, celle-ci variant selon les lieux et les époques, en fonction d'enjeux politiques, économiques, sociaux ou culturels, ignorés ou masqués. Les discours médicaux tenus au XIXe siècle sur l'hystérie offrent une parfaite illustration non seulement de l'infériorisation de la femme, mais également de la construction d'un discours racial et raciste plus général, visant à criminaliser les étrangers et les individus à la marge, analyse dont l'actualité est particulièrement frappante. 
Stéphanie Chapuis-Després analyse l'évolution du rôle des sages-femmes dans le SaintEmpire romain germanique aux XVIe et XVIIe siècles et souligne ce faisant un paradoxe : l'accouchement, qui est dans la pratique largement une affaire de femmes, est commenté et enseigné par les hommes qui investissent petit à petit le domaine de la gynécologie et de l'obstétrique. Ceci tient au fait que les sages-femmes sont en charge des naissances "normales", tandis que les médecins sont appelés pour les accouchements " pathologiques» dont ils tirent une plus grande gloire. On assiste en parallèle au renforcement progressif du contrôle et de la réglementation de l'activité des sages-femmes à qui l'on reproche leur présumé manque de formation. À l'heure où l'action des hommes sur le corps des femmes se généralise et où les pratiques obéissent à des règles inédites, parfois uniquement dictées par la pudeur, ce sont pourtant bien elles qui, par leur présence, garantissent la bienséance de ces nouveaux gestes médicaux, gestes qui, paradoxalement, sont parfois moins efficaces que ne l'étaient les leurs.

Sylvie Imparato-Prieur étudie le moment charnière dans l'Espagne du début du XIXe siècle lors duquel les chirurgiens, auteurs auparavant de traités d'obstétrique, étendent leur domaine de compétence, notamment par l'intermédiaire de cours, à la gynécologie et à la pédiatrie. Dans leurs travaux, le corps des femmes, comparé à celui des hommes, apparaît comme fondamentalement " déréglé », les menstrues faisant d'elles des êtres naturellement malades. Ces descriptions médicales font ainsi le jeu d'un discours moral qui réduit les femmes à leur fonction de procréatrices et justifie par la soi-disant fragilité de leur corps leur confinement au sein du foyer. Ces descriptions se mettent en outre au service de la volonté politique qui cherche à garantir à la collectivité des mères et donc des enfants en bonne santé. C'est toute l'ambivalence de ce discours pseudo-scientifique qui d'un côté exclut les femmes de l'espace public en glorifiant leur place au sein du ménage et de l'autre jette les bases des futures politiques de santé publique en contribuant à la connaissance de leurs pathologies propres.

Avec Emmanuelle Romanet-Da Fonseca, nous atteignons la seconde moitié du XIXe siècle, en France cette fois. Sa contribution s'attache au « duo explosif » formé par la syphilis et les nourrices dont le lait est vecteur de la maladie; elles peuvent ainsi infecter l'enfant et a contrario être contaminées par lui. Les progrès réalisés à propos des différents modes de transmission de la maladie sont à l'origine d'une modification de la perception de ce mal. Alors que la syphilis a longtemps été considérée comme synonyme de prostitution, l'« innocence » de certaines victimes favorise désormais la prise en charge des malades. Ce sera par exemple le cas à l'Hospice de l'Antiquaille de Lyon. L'article retrace par ailleurs comment, au fil du temps, le tabou autour de l'atteinte syphilitique est brisé, entre autres par le biais des procès intentés par les nourrices aux familles ayant caché la maladie de leur enfant au moment de les employer.

La deuxième partie du dossier, qui nous entraîne vers la Grèce antique puis moderne, se place à mi-chemin entre discours médical et discours littéraire.

18 Pascal Luccioni se penche sur les traités du médecin grec Galien (IIe siècle ap. JC) qui met en scène le diagnostic portant sur l'affection d'une de ses patientes due à ses peines de cœur. Or il s'agit là de la variation d'un épisode présent chez de nombreux auteurs de l'Antiquité qui relatent des cas de maladies d'amour similaires, à une différence près : le trouble y concerne non pas des femmes mais des jeunes hommes. La lecture comparative de ce corpus révèle d'une part que le «traitement » de la maladie d'amour 
est différent selon que l'on soit homme ou femme, autrement dit qu'il obéit à une grammaire genrée : appréciation/dépréciation d'un même comportement (en l'occurrence le silence), guérison/non-guérison, résistance par la force/par la passivité face à la tentation de l'amour interdit; elle montre d'autre part comment le genre, en tant que construction sociale et littéraire, impacte non seulement la mémoire du médecin/auteur, mais aussi celle de ses personnages et de ses lecteurs.

Sophie Coavoux aborde pour sa part la pathologisation de l'homosexualité dans la Grèce de la fin du XIXe siècle en se consacrant au poète Constantin Cavafy et à son œuvre. S'appuyant sur le concept foucaldien de scientia sexualis, elle montre comment la médecine (psychopathologie, psychiatrie) et la psychanalyse sont instrumentalisées par la société majoritaire, et en l'occurrence la critique littéraire, afin de dénoncer les comportements sexuels s'écartant de la norme hétérosexuelle et nourrir une rhétorique homophobe. L'originalité de Cavafy réside dans le fait que, contrairement à d'autres écrivains contemporains, il n'intériorise pas ce discours culpabilisant, mais l'intègre au contraire à sa poésie homoérotique pour mieux le détourner et le subvertir. L'ars erotica qu'il développe à cette occasion devient ainsi un contre-discours qui contribue à renverser celui de ceux qui présentent l'homosexualité comme pathologique et à interroger la compréhension hégémonique des catégories de " sain » et de «normal ».

Dans la troisième partie, les réflexions s'orientent plus résolument encore vers la représentation littéraire de la maladie, en particulier dans des textes en prose.

21 À travers l'œuvre de l'écrivaine Shi Pingmei, Jacqueline Estran éclaire le rôle majeur que joue la maladie pour de nombreuses artistes chinoises du XXe siècle qui, lors des périodes d'isolement liées à leurs affections, trouvent un espace de liberté propice à la création. Écriture féminine, maladie et corps souffrant entretiennent de ce fait une relation étroite dans leurs textes. Si les maux vécus sont l'un des ciments de la communauté formée par Shi Pingmei et son cercle d'amies, leur thématisation dans ses textes fictifs lui permet de rendre compte d'expériences douloureuses et également de s'inscrire dans une double filiation : celles de femmes faisant œuvre de leur souffrance physique et émotionnelle et celles de filles redonnant vie à leur mère au sein de leur œuvre. Les écrits autobiographiques attestent quant à eux de la quête existentielle et du parcours d'émancipation des protagonistes alter ego de l'auteure, comme lorsque l'une d'entre elles passe du statut de malade à celui de soignante en endossant le costume d'infirmière durant la guerre.

Ce sont des figures d'artistes hommes que la maladie (attaque cérébrale) ou l'addiction (alcoolisme) ont privés de leurs facultés créatrices qu'étudie pour sa part Catherine Delesalle-Nancey. Les deux nouvelles de F. Scott Fitzgerald choisies accordent toutefois elles aussi une grande place à la figure de la soignante, tantôt infirmière ou épouse. Or ces textes ne mettent pas au jour une répartition "genrée " des rôles, où la femme serait garante du care, tandis que l'homme en serait l'objet. Elle suit au contraire la piste d'une nouvelle «éthique du soin » qui transcende les catégories « hommes » et "femmes ». Elle les ancre dans une relation unissant deux sujets dans une même " économie du désir ", fondée sur la réciprocité d'un lien qui n'est dissymétrique qu’à première vue. C. Delesalle-Nancey complexifie par la suite la notion de "sujets pleins » en présentant soignant et soigné comme des êtres profondément " faillés ", habités à la fois par une pulsion de vie et de mort, ainsi que par un manque, moteur même du désir qui leur permet de se constituer en tant que sujet. 
Après les couples, la contribution de Thu Thuy Bui s'attache à étudier une relation père-fille, marquée par une rage destructrice et ce, à l'exemple de la littérature réputée difficile d'accès de Linda Lê. Le deuil du père libère chez sa fille, qui apparaît sous les traits du personnage tronqué de la "Manchote» dans Les Trois Parques, des troubles et des pulsions qui s'expriment non seulement dans la matérialité des mots, mais aussi à travers la rupture des normes de communication habituelles. Au-delà du père, c'est le double en soi et Dieu en personne qui sont pris pour cible. Parallèlement à l'analyse de la métaphore du feu ou de l'oiseau qui relient entre eux la fille, le père et la mort, l'étude montre comment l'écriture exerce une influence salutaire en aidant l'auteure à canaliser sa folie et à surmonter la crise qu'elle traverse suite aux différentes pertes subies : celle du pays d'origine d'abord, celle du «lecteur idéal » qu'était son père ensuite. formes de perte de repères, d'exil intérieur et de deuil. Elles découlent des séquelles laissées par un accident cérébral chez une écrivaine âgée d'une quarantaine d'années, dont on comprend aisément qu'elle est l'alter ego de l'auteure. Ici langue et souvenir ne déferlent pas, la patiente doit au contraire partir à leur conquête, au prix d'un long et douloureux travail de reconstruction mené en parallèle de sa convalescence physique. Le genre fournit une clé de lecture supplémentaire au travail de remémoration du personnage-narratrice qui revient sur sa trajectoire de citoyenne au sein de la République Démocratique Allemande (RDA) et sur ses amours troublées entre son mari Matthes et son amante Viola. À cette occasion, c'est également le potentiel salutogène de la maladie qui surgit, cette dernière donnant l'occasion d'une reconfiguration inattendue du langage et des significations. C'est sur cet élément positif que le dossier se clôt.

Ainsi les articles présentés abordent-ils la notion de maladie, ses spécificités selon qu'elle soit, par exemple, "féminine » ou "masculine», son statut en fonction des époques et des aires géographiques. Ils traitent des malades, mais également des remèdes apportés, de l'activité de care et des figures de soignant.e.s. Ils reviennent sur les perceptions qu'ont les collectivités de la "normalité » et de la «déviance », ainsi que sur l'impact de l'expérience de la maladie, et/ou des discours qui l'entourent, sur l'évolution personnelle et la construction identitaire des individus. Nous espérons qu'ils contribueront à affiner la compréhension du phénomène de la maladie et des relations qu'il entretient avec la question du genre dans l'Histoire et dans les arts.

\section{NOTES}

1. Nicole Edelman, Florence Rochefort (dir.), «Quand la médecine fait le genre », in Clio. Femmes, Genre, Histoire [En ligne], 37, 2013, mis en ligne le 19 juillet 2013. URL : http://clio.revues.org/ 10956. Dernière consultation : 5 mai 2017.

Transtext(e)s Transcultures 跨文本跨文化, 11 | 2016 